\title{
Dynamic response of the axial flow pump induced by the fluid excitation
}

\author{
Guofang Nan ${ }^{1, a}$, Yuanlu Zhang ${ }^{2, b}$, Eryun Chen ${ }^{3, c}$ \\ ${ }^{1}$ School of energy and power engineering, University of shanghai for science and technology, \\ Shanghai 200093, China; \\ ${ }^{2}$ School of energy and power engineering, University of shanghai for science and technology, \\ Shanghai 200093, China; \\ ${ }^{3}$ School of energy and power engineering, University of shanghai for science and technology, \\ Shanghai 200093, China; \\ angf@usst.edu.cn, bhangyuanluusst@163.com, chen_eryun@yahoo.com.cn
}

Keywords: axial flow pump; vibration; model dynamics; fluid excitation; responses

\begin{abstract}
The dynamic response for the axial flow pump induced by the fluid excitation is analyzed in this paper. The responses of the axial flow pump during the operation were mostly generated by the fluid excitation and motor excitation. The finite element model was accomplished in this article with consideration of the effect of the fluid excitation and the motor excitation forces. Based on the modal dynamics method, the analysis is carried out to study on the coupling responses under the two excitations. The results show that the vibration source includes the impeller frequency, the axial frequency and the frequency-doubled of the impeller; the response of the motor excitation occupies a leading position in the spectrogram. The research work is meaningful for solving the response induced by the flow excitation of the axial flow pumps.
\end{abstract}

\section{Introduction}

The axial flow pump is a type of pump which transports the flow along the axial direction by means of the impeller force. The axial flow pump is widely applied in large hydraulic engineering, drainage and irrigation. The vibrations and noises of the pump during its operation is associated with the pressure fluctuation. With the increasing standards of the vibrations and noises for the environmental protection, the issues of the vibrations and noises for the pump will attract more and more attention. It is necessary to study the vibrations induced by the flow excitation of the axial flow pump.

The vibrations and noises of the pump derive from the interior of the pump; and they are essentially related with the hydraulic pulsation of the inner pump [1-2]. The CFD method is usually applied to calculate the inner flow field of pump. Jeon $\mathrm{W} \mathrm{H} \mathrm{[3]} \mathrm{adopted} \mathrm{the} \mathrm{discrete} \mathrm{vortex} \mathrm{method} \mathrm{to}$ calculate the flow field under the effect of the centrifugal impeller and the wedge boundary. Langthjem M A et al [4-5] calculated the flow field distribution of the two dimensional pump and analyze the flow noise. Spence R [6-7] adopted the RANS method to calculate the flow field of inner pump and study on the influence of the load case on the fluctuation pressure. Much progress has been made in last decades. Li Yao-jun et al [8] simulated three-dimensional turbulent flows generated by an axial-flow pump equipped with an inducer by using the multiple reference frame approach. The predicated results of pump head and efficiency show reasonably good agreement with the experimental data. W. C. Zierke et al [9] carried out an experiment in order to provide a database for comparison with three-dimensional, turbulent flow computations, to evaluate engineering models, and to improve our physical understanding of many of the phenomena involved in this complex flow field. Durmus Kaya [10] studied the two different axial flow pump impellers with and without guide vanes; it is found that using guide vanes and regaining the tangential velocity energy of the pump improves the total pump efficiency about 3\%. P. Dupont et al [11] conducted an analysis of unsteady effects associated with rotor stator interactions in a vaned diffuser radial flow pump. Wang Fu-jun et al [12] performed the numerical simulations of 3D turbulent flow in a large-bore axial-flow pump coupled with half-elbow suction sump by using CFD approach Chen Hong-xun et al [13] applied the Ansys CFX software to analyze an axial-flow pump model with $0^{\circ}$ installation angle under steady and 
unsteady operating conditions; this work is of real significance in understanding accuracy of numerical simulation for axial-flow pump. Tremante Andres [14] establish the analysis of a numerical turbulent simulation of an axial pump cascade under two-phase flow presence of liquid and gas, coupled with the k turbulent model; and they carried out an experiment whose results show good agreement with the numerical results and confirm the existence of the gas pockets and the stratified flow in the blade passage.

The exciting forces which cause the vibrations of the axial flow pump derive from the flow excitation, the structural excitation and the motor excitation forces. The flow excitation force can be obtained from solving the unsteady equation Navier-Stokes; the structural excitation force was calculated by estimation of the unbalance level for the axial flow pump and the static deflection induced by the flow excitation force; the motor excitation force can be got from the experiments. In this paper, the numerical model of the axial flow pump induced by the flow excitation was developed to analyze the dynamic characteristics.

\section{Modelling and loads}

Importing the geometry model into the Abaqus and then meshing the components by using the type of C3D4 lead to the analytical model. The different types of element and dimension are used for the different parts in order to obtain the fine geometry features. The meshing quality of the elements is checked to get a good quality and the parts will be remeshed if their quality does not meet the requirements until the quality is good enough for calculation. The parameters used are as follows: Modulus of elasticity $E=210 \mathrm{GPa}$, density $\rho=7850 \mathrm{Kg} / \mathrm{m}^{3}$, Poisson ratio $\mu=0.3$. After calculating the free mode of the components and comparing the results with that of experiments, it is found that they agree well, as seen in Table 1. Thereby, this comparison and correction can validate the accuracy of the whole model.

Table 1 Comparison of the FE results with the experiment results for the first natural frequency

\begin{tabular}{cccc}
\hline Component & $\begin{array}{c}\text { Experimen } \\
\mathrm{t}\end{array}$ & FEM & $1^{\text {st }}$ Frequency error (\%) \\
\hline Pump body & 423 & 433 & 2.4 \\
Guide vane & 985 & 962 & -2.3 \\
Impeller chamber & 912 & 933 & 2.3 \\
shaft & 249 & 246 & -1.2 \\
\hline
\end{tabular}

The connections between the two components in axial flow pump mainly include the bolting, the shaft coupling and so on. The springs with the different stiffness values which come from the experiments are applied to simulate the connections in this paper. The connectors between the inlet/outlet pipes with the exterior are also simulated to be the springs with a specific stiffness values; and the type of springs is connect point to ground. The springs are built with the different types and stiffness with consideration of the stiffness in three components in order to be closer to reality. After assembling the components and creating the connections, the analytical model was accomplished, as shown in Fig. 1, where A is the analytical point. 


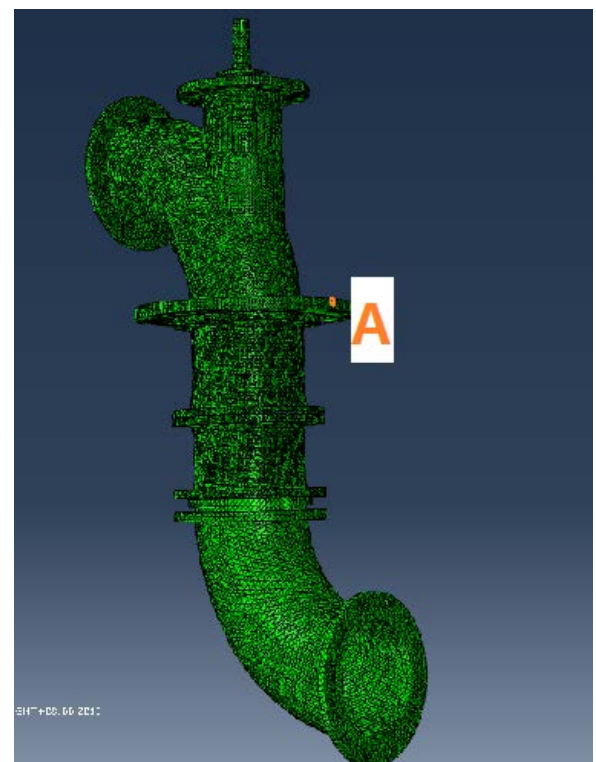

Fig. 1 The Finite element model of the whole axial flow pump

The modal dynamics method is adopted in this paper to calculate the responses. This method applies the modal superposition method to solve the transient responses of the linear system. The characteristic modes should be extracted before this method based on the characteristic modes is applied. The method is applied to make the time-domain analysis of a linear system, where the excitation is a function of time and there is an assumption that the amplitude in every incremental time step is linear under the determined excitation. In this paper, the exciting forces are the fluid excitation force which was obtained by the numerical simulation, and the motor excitation force which was got from the experiments. These two excitation forces are exerted on the analytical model to submit calculation.

\section{Results and analysis}

The response on the pedestal of the pump under the effect of the fluid excitation and the motor excitation will be investigated in this section. The constrained modes of the axial flow pump which are connected at the inlet/outlet pipes and the pedestal are calculated before the responses are calculated. Appling the Spring (connect point to ground), the feet of the machine, the inlet and the outlet are joined by Springs to the ground. The constrained modes are calculated by using the Lanczos Method; the results are seen in Table 2. The analytical point on the pedestal A is selected as the analytical point of the axial flow pump, as seen in Fig. 1. Exerted by the flow excitation and the motor excitation, the axial flow pump system was submitted into calculation by using the modal dynamics method. Figure 2(a)-(c) are the displacement frequency response, the velocity frequency response and the acceleration frequency response, respectively. It can be seen that the response of the motor excitation occupies a leading position in the frequency response figures; the resonant responses are excited at the $1 \mathrm{BPF}$ and $1074 \mathrm{~Hz}$. Figure 2(d) is the distribution of the acceleration level; the acceleration level at the $1 \mathrm{BPF}$ is $87 \mathrm{~dB}$ and the acceleration level at $1074 \mathrm{~Hz}$ is $126 \mathrm{~dB}$. The total vibration level for $10-315 \mathrm{~Hz}$ is $102.5 \mathrm{~dB}$; and the total vibration level for $10-2000 \mathrm{~Hz}$ is $136 \mathrm{~dB}$. 


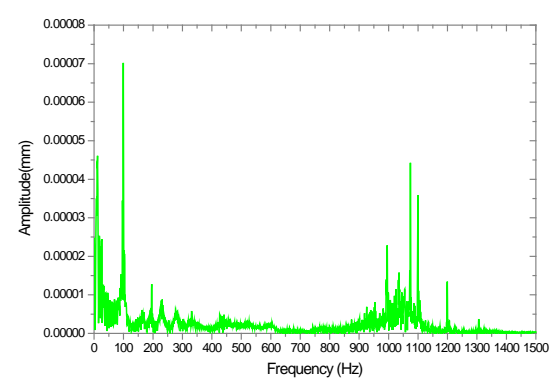

(a)

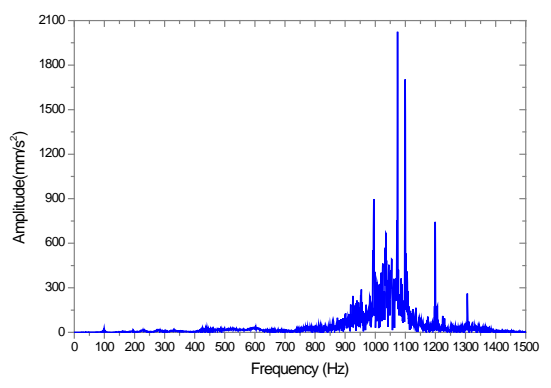

(c)

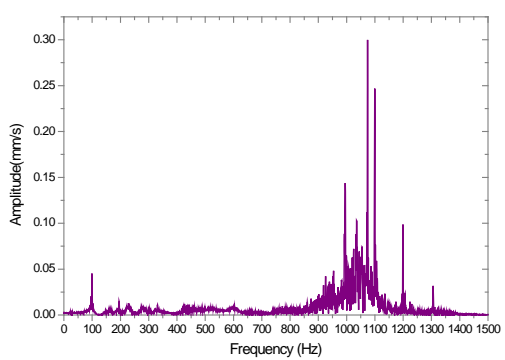

(b)

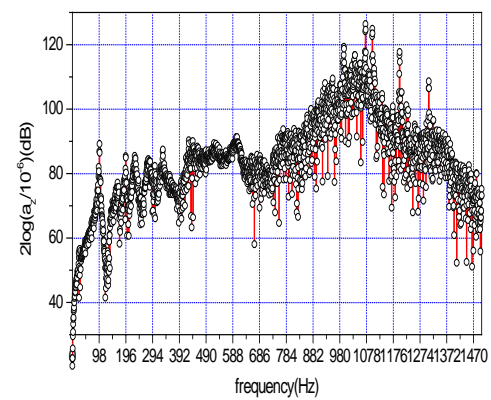

(d)

Fig. 2 The responses of the pedestal a) displacement; b) velocity; c) acceleration; d) distribution of the acceleration level

Table 2 The main constrained modes (constrained on the inlet/outlet and the feet of the machine)

\begin{tabular}{cc}
\hline Order & Frequency $(\mathrm{Hz})$ \\
\hline 1 & 27.324 \\
2 & 29.857 \\
3 & 99.850 \\
4 & 139.01 \\
5 & 167.46 \\
6 & 175.61 \\
7 & 193.13 \\
8 & 213.33 \\
\hline
\end{tabular}

It can be seen from Fig. 2(d) that there are three notable frequencies, i.e. $84.61 \mathrm{~dB}(24.17 \mathrm{~Hz})$ that is the frequencies of axial, $91.71 \mathrm{~dB}(96.67 \mathrm{~Hz})$ that is the frequency of the impeller and $89.86 \mathrm{~dB}$ $(193.33 \mathrm{~Hz}$ ) that is the frequency-doubled.

\section{Conclusions}

Based on the modal dynamics method, the dynamic response for the axial flow pump induced by the fluid excitation is analyzed in this paper, the main conclusions are as follows:

1) The resonant responses are excited at the $1 \mathrm{BPF}$ and $1074 \mathrm{~Hz}$; the acceleration level at the $1 \mathrm{BPF}$ is $87 \mathrm{~dB}$ and the acceleration level at $1074 \mathrm{~Hz}$ is $126 \mathrm{~dB}$. The total vibration level for $10-315 \mathrm{~Hz}$ is $102.5 \mathrm{~dB}$; and the total vibration level for $10-2000 \mathrm{~Hz}$ is $136 \mathrm{~dB}$.

2) There are three notable frequencies, i.e. $84.61 \mathrm{~dB}(24.17 \mathrm{~Hz})$ that is the frequencies of axial, 91.71 $\mathrm{dB}(96.67 \mathrm{~Hz})$ that is the frequency of the impeller and $89.86 \mathrm{~dB}(193.33 \mathrm{~Hz})$ that is the frequency-doubled.

3) The vibration spectrum includes the spectrum of the impeller frequency and the spectrum of the axial frequency which was caused by the impeller frequency and the frequency-doubled of the impeller; the response of the motor excitation occupies a leading position in spectrogram. 


\section{Acknowledgement}

The authors are grateful to the financial support of the National Science Foundation of China

(Granted number: 51305267)

\section{References}

[1] Johann Friedrich Gülich: Centrifugal Pumps. ( Secondedition) [M] . Springer, 2010.

[2] Bolton N: Noise generation in pumps. Noise in Fluid Machinery [M] . ImechE Seminar Publication, London, UK, March, 1999.

[3] Jeon W H, Lee D J: A numerical study on the flow and sound fields of centrifugal impeller located near a wedge $[\mathrm{J}]$. Journal of Sound and Vibration, 266 ( 2003) 785-804.

[4] Langthjem M A, Olhoff N: A numerical study of flow-induced noise in a two-dimensional centrifugal pump. Part I. Hydrodynamics [J] . Journal of Fluids and Structures, 2004, 19: 349-368.

[5] Langthjem M A, Olhoff N: A numerical study of flow-induced noise in a two-dimensional centrifugal pump. Part II. Hydroacoustics [J] . Journal of Fluids and Structures, 2004, 19: 369-386.

[6] Spence R, Amaral-Teixeira J: Investigation into pressure pulsations in a centrifugal pump using numerical methods supported by industrial tests $[\mathrm{J}]$. Computers \& Fluids, 2008, 37: 690-704.

[7] Spence R, Amaral-Teixeira J: A CFD parametric study of geometrical variations on the pressure pulsations and performance characteristics of a centrifugal pump $[\mathrm{J}]$. Computers \& Fluids, 2009, 38: 1243-1257.

[8] Li Yao-jun, Wang Fu-jun: Numerical Investigation of performance of an axial-flow pump with inducer, Journal of Hydrodynamics, 2007,19(6):705-711

[9] W. C. Zierke, W. A. Straka and P. D. Taylor: An Experimental Investigation of the Flow Through an Axial-Flow Pump, J. Fluids Eng 117(3), 485-490

[10]Durmus Kaya: Experimental study on regaining the tangential velocity energy of axial flow pump, Energy Conversion and Management, Volume 44, Issue 11, July 2003, Pages 1817-1829

[11]P. Dupont, G. Caignaert, G. Bois and T. Schneider: Rotor-Stator Interactions in a Vaned Diffuser Radial Flow Pump, ASME 2005 Fluids Engineering Division Summer Meeting Volume 1: Symposia, Parts A and B Houston, Texas, USA, June 19-23, 2005 Conference Sponsors: Fluids Engineering Division ISBN: 0-7918-4198-7

[12] Wang Fu-jun, Li Yao-jun, Cong Guo-hui, Wang Wen-e, Wang Hai-song: CFD simulation of 3D flow in large-bore axial-flow pump with half-elbow suction sump, Journal of hydrodynamics,2006, 18(2):243-247

[13] Chen Hong-xun, Zhu Bin: Analysis of numerical calculation on an axial-flow pump model with 0 installation angle [J]. Chinese Journal of Hydrodynamics, 2009, 24(4): 480-484Tremante Andres, Moreno Nathaly, Noguera Ricardo et al. Numerical turbulent simulation of the two-phase flow (liquid/gas) through a cascade of an axial pump[A].

[14] Proceedings of the 2001 ASME Fluids Engineering Division Summer Meeting[C]. New Orleans, USA, 2001, 2: 355-36 